\title{
Recommandations
}

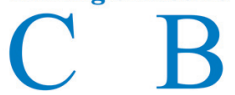

\section{Gestion péri-opératoire des patients traités par antithrombotiques en chirurgie orale. Recommandations}

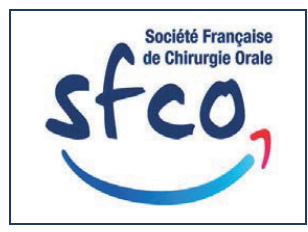

En collaboration avec :
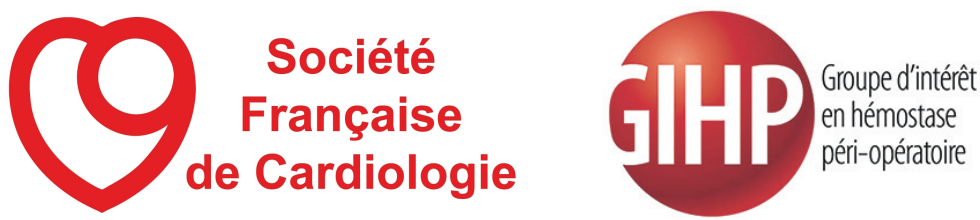

\section{Groupe de travail}

Pr Jean-Christophe FRICAIN (Chirurgie orale, Bordeaux)

\section{Président}

DrCédric MAUPRIVEZ (Chirurgie orale, Paris) Rapporteur

Dr Pierre GANGLOFF (Chirurgie orale, Metz-Thionville)

Dr Sylvie BOISRAME (Chirurgie orale, Brest)

Dr Patrick LIMBOUR (Chirurgie orale, Rennes)

PrPierre ALBALADEJO (Anesthésie-réanimation, Grenoble)

Dr Annick ANKRI (Hématologie, Paris)

Dr Fabrice CAMPANA (Chirurgie orale, Marseille)

Dr Sarah COUSTY (Chirurgie orale, Toulouse)

Pr Vianney DESCROIX (Pharmacologie, Paris)

Mr Antoine GOBILLARD (Association Malades)

Pr Bernard IUNG (Cardiologie, Paris)

PrIsabelle MAHE(MédecineInterne, Cardiologie, Colombes)

PrJean-PaulMENINGAUD (Chirurgie maxillo-faciale, Créteil)

Dr Jean-Jacques MONSUEZ (Cardiologie, Sevran)

PrEricPAUTAS(MédecineInterne, Gériatrie,Ivry-sur-Seine)

Pr Jacky SAMSON (Stomatologie, Genève)

\section{Groupe de lecture}

Dr Alp ALANTAR (Chirurgie orale, Nanterre)

Dr Ygal ATTAL (Médecine générale, Chambéry)

Dr Anne-Gaelle BODARD (Chirurgie orale, Lyon)

Dr Vanessa BAAROUN (Chirurgie orale, Paris)

Dr Jean-Hugues CATHERINE (Chirurgie orale, Marseille)

Dr Rémi CURIEN (Chirurgie orale, Maxéville)

Dr Christophe DESCHAUMES (Chirurgie orale, Clermont-Ferrand)
DrÉmilie DOCHE (Neurologie, Unité Neurovasculaire, Marseille)

Dr Célia DUMAS (Médecine générale, Toulouse)

Dr Anne-Laure EJEIL (Odontologie, Paris)

Dr Philippe GUIBELLINO (Médecine d'urgence, Marseille)

Dr Michel GUYOT (Chirurgie orale, Saint-Herblain)

Dr Hilal HAFIAN (Chirurgie orale, Reims)

Dr Emannuelle HERLEM (Médecine générale, Pharmacovigilance, Reims)

Dr Philippe JAUFFRION (Cardiologie, Villejuif)

Dr Nicolas JOVENIN (Cancérologie, Saint-Dizier)

Pr Karine LACUT (Thérapeutique, Brest)

Dr Patrick LARRAS (Chirurgie orale, Dijon)

Dr Florian LAURENT (Odontologie, Igny)

Pr Benoît LEFEVRE (Chirurgie orale, Reims)

Dr Hubert LE HETET (Anesthésie-réanimation, Cesson-Sévigné)

Pr Philippe LESCLOUS (Chirurgie orale, Nantes)

Dr Raphaël LOPEZ (Chirurgie maxillo-faciale, Toulouse)

Pr Louis MAMAN (Chirurgie orale, Ivry-sur-seine)

Dr Jean-Pierre MARIOTTINI (Odontologie, Nice)

Dr Hervé MOIZAN (Chirurgie orale, Rouen)

Pr Jean-Louis MONTASTRUC (Pharmacologie, Toulouse)

Dr Laurent NAWROCHI (Chirurgie orale, Lille)

Dr Laurence NOËL-FURON (Chirurgie orale, Rethel)

Dr William OHANA (Odontologie, Strasbourg)

Pr Guillaume PENEL (Chirurgie orale, Lille)

Dr Omar RAZOUK (Chirurgie orale, Paris)

Dr Marouane TALBI (Chirurgie maxillo-faciale, Metz-Thionville) Dr Nicolas WEISS (Neurologie, Unité de Réanimation neurologique, Paris) 


\section{Contexte d'élaboration}

Ces dernières années, la thérapeutique antithrombotique a considérablement évolué avec l'arrivée de nouveaux médicaments. Deux nouveaux agents antiplaquettaires sont utilisés depuis 2010, le prasugrel et le ticagrelor. Trois nouveaux anticoagulants oraux ayant une action sélective et spécifique sur les facteurs II et $X$ activés, appelés anticoagulants oraux directs ont été mis sur le marché : le dabigatran en 2008, le rivaroxaban en 2009 et l'apixaban en 2012. Pour chacune de ces molécules, des progrès thérapeutiques sont avancés : une meilleure efficacité pour les nouveaux antiplaquettaires avec une diminution du nombre de non répondeurs, l'existence d'une marge thérapeutique large et l'absence de surveillance biologique pour les nouveaux anticoagulants. En revanche, il n'existe aucun antidote spécifique actuellement commercialisé pour ces nouveaux anticoagulants.

Enfin, de plus en plus de patients bénéficient d'une bithérapie antithrombotique (combinaison de deux antiplaquettaires ou association d'un antiplaquettaire et d'un anticoagulant oral).
L'arrivée de ces nouvelles molécules et de nouveaux protocoles de prise en charge et de prévention des accidents thromboemboliques a amené à repenser l'approche du patient traité par antithrombotique en chirurgie orale. C'est la raison pour laquelle la Société Française de Chirurgie Orale a engagé en 2013 une réactualisation de ces recommandations concernant la prise en charge des patients bénéficiant d'une thérapeutique antiplaquettaire et/ou anticoagulante en chirurgie orale (Recommandations SFMbCb 2005, 2006).

\section{Objectifs de ces recommandations :}

1- Définir, pour chacune des familles d'antithrombotiques, le risque hémorragique en cas de maintien de la thérapeutique antithrombotique en fonction des différentes interventions et/ou gestes invasifs de chirurgie orale ;

2- Formaliser la gestion péri-opératoire des patients traités par antithrombotiques en cas de chirurgie orale.

\section{Grade des recommandations}

\begin{tabular}{|ll|}
\hline A & $\begin{array}{l}\text { Preuve scientifique établie } \\
\text { Fondée sur des études de fort niveau de preuve (niveau de preuve 1): essais comparatifs randomisés } \\
\text { de forte puissance et sans biais majeur ou méta-analyses d'essais comparatifs randomisés, } \\
\text { analyses de décisions basées sur des études bien menées. }\end{array}$ \\
\hline B & $\begin{array}{l}\text { Présomption scientifique } \\
\text { Fondée sur une présomption scientifique fournie par des études de niveau intermédiaire de preuve } \\
\text { (niveau de preuve 2), comme des essais comparatifs randomisés de faible puissance, des études } \\
\text { comparatives non randomisées bien menées, des études de cohorte. }\end{array}$ \\
\hline C & $\begin{array}{l}\text { Faible niveau de preuve } \\
\text { Fondée sur des études de moindre niveau de preuve, comme des études cas-témoins (niveau de } \\
\text { preuve 3), des études rétrospectives, des séries de cas, des études comparatives comportant des } \\
\text { biais importants (niveau de preuve 4). }\end{array}$ \\
\hline AE & $\begin{array}{l}\text { Avis d'experts } \\
\text { En l'absence d'études, les recommandations sont fondées sur un accord entre experts du groupe } \\
\text { de travail, après consultation du groupe de lecture. L'absence de gradation ne signifie pas que les } \\
\text { recommandations ne sont pas pertinentes et utiles. Elle doit, en revanche, inciter à engager des } \\
\text { études complémentaires. }\end{array}$ \\
\hline
\end{tabular}




\section{Recommandations}

\section{Généralités pour l'ensemble des antithrombotiques}

1- L'évaluation du risque hémorragique chirurgical est du ressort du chirurgien. Cette évaluation repose avant tout sur l'interrogatoire médical et l'examen clinique pré-opératoire $B$.

2- L'évaluation du risque thrombotique est du ressort du médecin prescripteur de l'antithrombotique. Son évaluation est indispensable en cas de chirurgie orale à risque hémorragique élevé afin de définir la conduite à tenir visà-vis de la thérapeutique antithrombotique (maintien, arrêt temporaire avec ou sans traitement substitutif) AE.

3- En cas de chirurgie orale à faible risque hémorragique comme les avulsions dentaires $A$, les interventions de chirurgie parodontale ou la pose d'implants dentaires $A E$, la poursuite du traitement antithrombotique est recommandée.

4- Les facteurs favorisants les accidents hémorragiques chez les patients sous antithrombotiques sont multiples et doivent conduire à une vigilance accrue sans être considérés comme des contre-indications AE.

5- Afin de limiter le risque hémorragique et de faciliter la prise en charge d'une éventuelle complication hémorragique, il est préférable que les interventions chirurgicales soient programmées le matin et en début de semaine. En cas de chirurgies multiples, une approche segmentaire diminue ce risque $A E$.

6- La prise en charge des patients traités par antithrombotiques peut se faire en pratique de ville, sous réserve de la coopération du patient et de la proximité d'une structure hospitalière ( $<50 \mathrm{~km}$ ou $<1$ heure) ou d'une filière de soins pré-établie compétente dans la gestion de ce type de patient (ex : cabinet de chirurgie orale) AE.

7- Les techniques d'hémostase locale sont indispensables et systématiquement associées aux actes de chirurgie orale $A$.

8- La continuité des soins doit être impérativement assurée. Tout patient traité par antithrombotique(s) ayant une complication hémorragique postopératoire doit pouvoir contacter un praticien compétent dans la gestion de ce type de complication dans le cadre d'un réseau de soins ou le cas échéant un service hospitalier d'0dontologie, de Chirurgie Orale, de Stomatologie et/ou de Chirurgie Maxillo-Faciale d'astreinte AE.

9- En cas de chirurgie à risque hémorragique élevé (cf annexe 1), l'avis du médecin prescripteur de l'antithrombotique est indispensable afin d'évaluer le risque thrombotique. En cas de risque thrombotique faible, un arrêt temporaire du traitement antithrombotique et une prise en charge en pratique de ville peuvent être discutés. En cas de risque thrombotique élevé, une prise en charge hospitalière est recommandée $A E$.

10- En cas d'hémorragie postopératoire non contrôlable par une compression mécanique, la règle est la reprise chirurgicale de l'hémostase. Après anesthésie locale, curetage du caillot ou simple décaillottage par aspiration, la plaie opératoire est révisée, puis l'hémostase locale reprise selon les procédures habituelles. Les conseils post-opératoires sont renouvelés. En cas de saignement persistant malgré la reprise de l'hémostase, le patient doit être hospitalisé jusqu'à ce que la situation soit maîtrisée $A$.

\section{Spécificités de la prise en charge d'un patient traité par AAP}

11- La poursuite d'une monothérapie par agents antiplaquettaires (AAP) est recommandée pour tout acte de chirurgie orale quelque soit le risque hémorragique associé (A pour les avulsions dentaires et AE pour la pose d'implants dentaires et tous les autres actes).

12- La poursuite d'une bithérapie par AAP est recommandée en cas de chirurgie orale à faible risque hémorragique ( $B$ pour les avulsions dentaires et $A E$ pour la pose d'implants et tous les autres actes). En cas de chirurgie orale à risque hémorragique élevé (cf annexe 1), l'avis du médecin prescripteur est obligatoire afin de définir la stratégie thérapeutique optimale $A E$.

13- À ce jour, il n'existe pas d'examen biologique prédictif du risque hémorragique chirurgical lié à la prise d'un AAP A.

\section{Spécificités de la prise en charge d'un patient traité par AVK}

14- La poursuite du traitement par antivitamines K (AVK) est recommandée en cas de chirurgie orale à faible risque hémorragique ( $\mathrm{A}$ pour les avulsions dentaires, B pour la pose d'implants et AE pour tous les autres actes). La valeur de l'INR doit être mesurée idéalement dans les 24 heures, au maximum dans les 72 heures précédant l'intervention chirurgicale. Elle doit être stable et inférieure à $4 \mathrm{~A}$. En cas de surdosage (INR supérieur ou égal à 4) ou d'INR instable, l'intervention chirurgicale doit être reportée et le médecin prescripteur informé. Des mesures correctrives doivent être instaurées sans délai par le médecin prescripteur afin de ramener l'INR en zone thérapeutique.

15- En cas de chirurgie orale à risque hémorragique élevé (cf annexe 1), l'avis du médecin prescripteur est indispensable afin d'évaluer le risque thrombotique. En cas de risque thrombotique faible, une fenêtre thérapeutique de 48 à 72 heures peut être proposée par le médecin prescripteur. Les AVK doivent être arrêtés 24 à 48 heures avant l'intervention chirurgicale (24 heures pour l'acénocoumarol et 48 heures pour la coumadine ou la fluindione), l'INR contrôlé en préopératoire avec pour objectif une valeur inférieure ou égale à 1,5 et une reprise des AVK dès que possible au plus tard dans les 24 à 48 heures postopératoires 
C. Dans les rares cas de chirurgie à risque hémorragique élevé chez un patient présentant un risque thrombotique élevé, l'instauration en milieu hospitalier d'un relais pré et postopératoire du traitement AVK par une héparine (héparine de bas poids moléculaire ou non fractionnée) à dose curative est possible mais doit rester exceptionnelle $C$.

16- Les prescriptions médicamenteuses pré et postopératoires peuvent induire des interactions médicamenteuses avec les AVK. Pour le contrôle de la douleur, le paracétamol et les dérivés opiacés peuvent être prescrits. L'aspirine et les anti-inflammatoires non stéroïdiens (AINS) sont contre-indiqués car ils exposent à un risque hémorragique majoré lorsqu'ils sont associés aux AVK A. Pour le contrôle du risque infectieux, des cas sporadiques d'augmentation de l'INR ont été rapportés après la prise d'amoxicilline seule ou associée à l'acide clavulanique, de clindamycine, de métronidazole, tous les macrolides à l'exception de la spiramycine. Les patients doivent en être informés et un contrôle de l'INR dans les jours suivant la prescription est recommandé C. À noter qu'une antibioprophylaxie $(2 \mathrm{~g}$ d'amoxicilline ou $600 \mathrm{mg}$ de clindamycine, 1 heure avant l'intervention) ne modifie pas la valeur de l'INR.

\section{Spécificités de la prise en charge d'un patient traité par AOD}

17- La poursuite du traitement par anticoagulants oraux directs (AOD) est recommandée en cas de chirurgie orale à faible risque hémorragique : avulsions dentaires, pose d'implants et AE pour tous les autres actes.

18- En cas de chirurgie orale à risque hémorragique élevé (cf annexe 1), l'avis du médecin prescripteur est indispensable afin d'évaluer le risque thrombotique. En cas de risque thrombotique faible, les AOD peuvent être arrêtés par le médecin la veille de l'intervention et repris le lendemain de l'intervention (fenêtre thérapeutique de 48h) AE. Dans les rares cas de chirurgie à risque hémorragique élevé chez un patient présentant un risque thrombotique élevé, un arrêt plus prolongé du traitement par $\mathrm{AOD}$ avec un relais par une héparine (héparine de bas poids moléculaire ou héparine non fractionnée) en milieu hospitalier est possible mais doit rester exceptionnel $\mathrm{AE}$.

19- À ce jour, il n'existe pas d'examen biologique prédictif du risque hémorragique chirurgical lié à la prise d'un AOD A.

20- La prescription de clarithromycine chez un patient traité par AOD doit être évitée $C$.

\section{Spécificités de la prise en charge d'un patient traité par héparine}

21- La poursuite du traitement par une héparine (héparine de bas poids moléculaire ou héparine non fractionnée) est recommandée en cas de chirurgie orale à faible risque hémorragique

( $C$ pour les avulsions dentaires, AE pour la pose d'implants et pour tous les autres actes).

22- En cas de chirurgie orale à risque hémorragique élevé (cf annexe 1) les héparines peuvent être arrêtées par le médecin prescripteur avant l'intervention ( 6 à 8 heures avant l'intervention pour les héparines non fractionnées et 24 heures avant pour les héparines de bas poids moléculaire) afin de limiter le risque de saignement péri-opératoire $C$.

23- En cas d'arrêt pré-opératoire d'une héparinothérapie, la prescription pré-opératoire d'un temps de céphaline activé (TCA) ou d'une activité anti Xa n'est pas nécessaire A. 
Annexe I. Stratification du risque hémorragique en fonction du type de chirurgie et mesures préventives.

\begin{tabular}{|c|c|}
\hline Type de chirurgies et actes invasifs & Mesures préventives des complications hémorragiques \\
\hline \multicolumn{2}{|l|}{ Actes sans risque hémorragique } \\
\hline $\begin{array}{l}\text { - Anesthésie locale } \\
\text { - Détartrage }\end{array}$ & - Hémostase mécanique simple par pression \\
\hline \multicolumn{2}{|l|}{ Chirurgies et actes à faible risque hémorragique } \\
\hline \multicolumn{2}{|c|}{$\begin{array}{l}\text { (Chirurgies pour lesquelles une hémorragie extériorisée est facilement contrôlable par une hémostase chirurgicale } \\
\text { conventionnelle *) }\end{array}$} \\
\hline $\begin{array}{l}\text { - Avulsion simple } \\
\text { - Avulsions multiples dans } 1 \text { même quadrant } \\
\text { - Chirurgie endodontique, périapicale, énucléation de kystes } \\
\text { et tumeurs bénignes (lésion }<3 \mathrm{~cm} \text { ) } \\
\text { - Chirurgie muco-gingivale (hors greffe gingivale avec } \\
\text { prélèvement palatin) } \\
\text { - Chirurgie pré-orthodontique d'une dent enclavée, incluse } \\
\text { - Implant unitaire } \\
\text { - Dégagement implant(s) (pilier cicatrisation) } \\
\text { - Biopsie-exérèse muqueuse orale }(\leq 1 \mathrm{~cm})\end{array}$ & $\begin{array}{l}\text { - Mesure d'hygiène bucco-dentaire et détartrage } \\
\text { - Hémostase chirurgicale conventionnelle } \\
\text { - Acide tranexamique }\end{array}$ \\
\hline \multicolumn{2}{|c|}{ Chirurgies et actes invasifs à risque hémorragique élevé } \\
\hline \multicolumn{2}{|c|}{$\begin{array}{l}\text { (Chirurgies pour lesquelles des pertes sanguines significatives et/ou transfusions plaquettaires sont rapportées dans } \\
\text { la littérature, interventions d'une durée opératoire > } 1 \text { heure, interventions critiques par leurs localisations (sinus } \\
\text { maxillaire, plancher buccal) et/ou difficilement contrôlables par une hémostase chirurgicale conventionnelle *). }\end{array}$} \\
\hline $\begin{array}{l}\text { - Avulsions multiples dans plusieurs quadrants } \\
\text { - Avulsion de dent(s) incluse(s) } \\
\text { - Implants multiples dans plusieurs quadrants } \\
\text { - Elévation du sinus (voie crestale, voie latérale) } \\
\text { - Greffes osseuses d'apposition (en onlay) } \\
\text { - Greffe osseuse particulaire et régénération osseuse guidée } \\
\text { - Chirurgie des tissus mous (lithiase salivaire) } \\
\text { - Chirurgie endodontique, périapicale, énucléation de kystes } \\
\text { et tumeurs bénignes (lésion > } 3 \mathrm{~cm} \text { ) } \\
\text { - Fermeture d'une communication bucco-sinusienne } \\
\text { - Exérèse des pseudotumeurs et tumeurs bénignes de la } \\
\text { muqueuse buccale }(>1 \mathrm{~cm})\end{array}$ & $\begin{array}{l}\text { - Même mesure préventive que pour une chirurgie à } \\
\text { risque hémorragique faible à modéré } \\
\text { - Médicaments dérivés du sang, à base de } \\
\text { fibrinogène et thrombine humains } \\
\text { - Electrocoagulation mono et bipolaire } \\
\text { - Privilégier les chirurgies mini-invasives (flapless et } \\
\text { chirurgie implantaire guidée, abord du sinus par } \\
\text { voie crestale...) } \\
\text { - Imagerie 3D préopératoire (sinus, région } \\
\text { symphysaire) en cas de pose d'implant }\end{array}$ \\
\hline
\end{tabular}


Annexe I. Suite.

\section{Gestes déconseillés}

- Bloc du nerf alvéolaire inférieur: déconseillé

- Greffe autologue : déconseillée en raison d'un site de prélèvement supplémentaire, privilégier les greffons hétérologues et synthétiques

\section{Gestes contre-indiqués}

- Tous les gestes contre-indiqués en cas de risque d'endocardite infectieuse associé

- Tous les gestes présentant un risque hémorragique dans le cas où le plateau technique à la disposition du chirurgien est insuffisant

- Bloc du nerf alvéolaire inférieur bilatéral : risque d’hématome latéropharyngé bilatéral et de dyspnée

- Prélèvement symphysaire : risque d'hématome du plancher buccal et de dyspnée

- Greffe gingivale avec prélèvement palatin : risque de lésion de l'artère palatine

* hémostase chirurgicale conventionnelle : hémostase mécanique (pression+ sutures) \pm hémostatiques locaux résorbables (éponges collagène ou gélatine, gaze de cellulose) \pm colle synthétique (colle de cyanoacrylate).

Facteurs majorant le risque hémorragique opératoire : décollement muco-périosté au délà de la ligne muco-gingivale, décollement lingual, avulsion(s) en zone inflammatoire, parodonte amoindri, durée opératoire $>1 \mathrm{~h}$ (perte sanguine significative),

Localisations critiques : plancher buccal, symphyse mentonnière, sinus maxillaire 
Annexe II. Algorithme systématique de prise en charge des patients sous agents antiplaquettaires (AAP) en chirugie orale.

\section{Patients sous agents antiplaquettaires}

\section{MONOTHÉRAPIE}

Aspirine KARDEGICR Clopidogrel PLAVIX®B
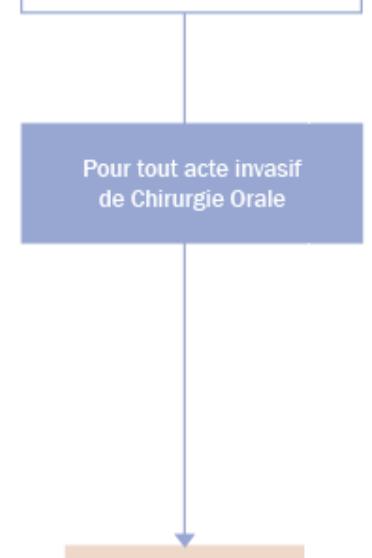

Pas d'arrêt

Prise en charge

en pratique de ville

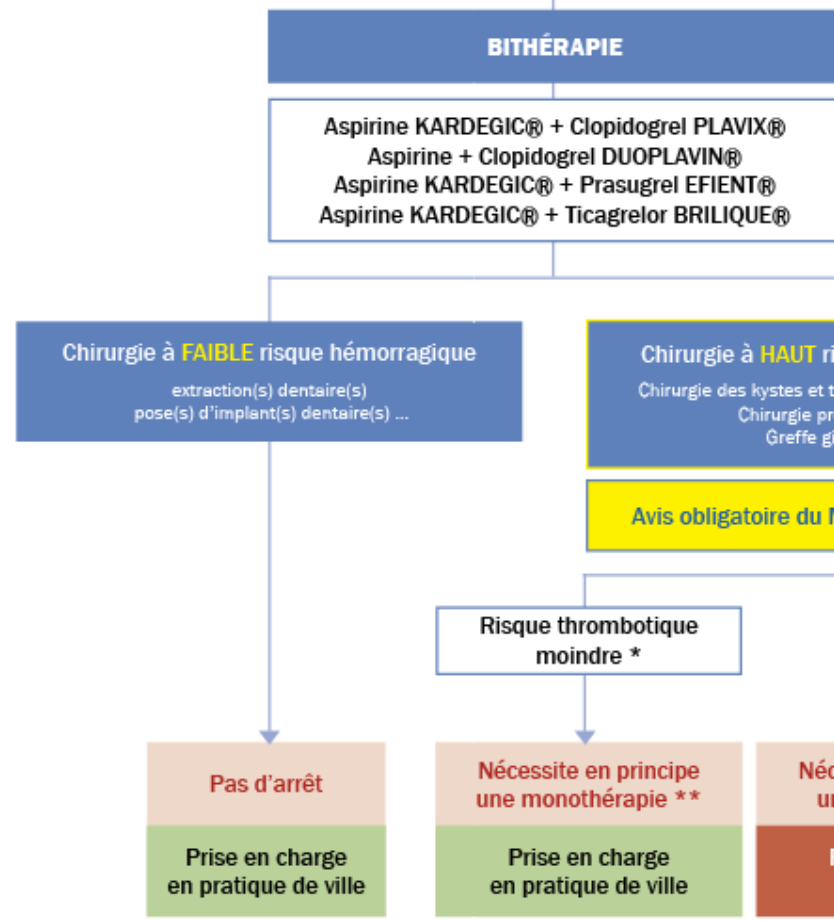

\section{BITHÉRAPIE}

Aspirine KARDEGIC(R) + Clopidogrel PLAVIX

Aspirine + Clopidogrel DUOPLAVIN(B)

spirine KARDEGIC(R) + Prasugrel EFIENTR

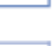

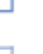

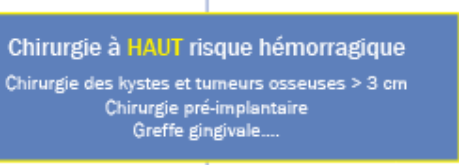

Avis obligatoire du Médecin prescripteur

* Détermination du niveau de risque thrombotique site consultable : www.has-sante.fr , * Recommandations : Antiagregants-plaquettaires : prise en compte des risques thrombotiques et hémorragique pour les gestes percutanés chez le coronarien * (HAS novembre 2013).

* * Monothérapie : poursuivre la prescription d'aspirine, délai d'interruption: clopidogrel : 5 jours, prasugrel : 7 jours, ticagrelor 3 à 5 jours. 
Annexe III. Algorithme systématique de prise en charge des patients sous antivitamine K (AVK) en chirugie orale.

\section{Patients sous antivitamines $K$}

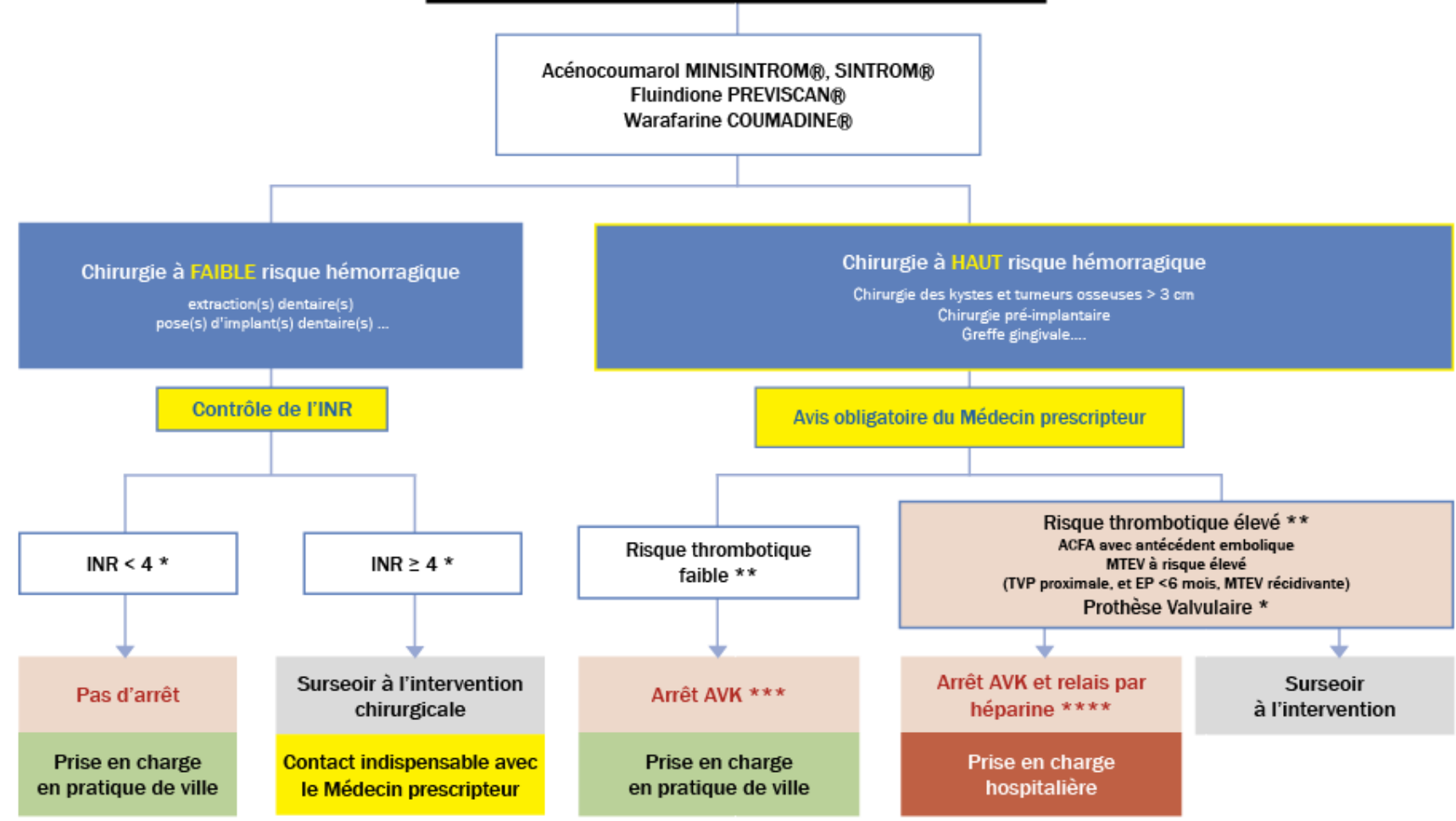

* Chez les patients porteurs de prothèses valvulaires, la pose d'implant, la chirurgie pré-implantaires et parodontale sont contre-indiqués.

** Détermination du niveau de risque thrombotique : site consultable : www. has-sante.fr * Recommandations : Prise en charge des surdosages en antivitamines $\mathrm{K}$, des situations à risque hémorragique et des accidents hémorragiques chez les patients traités par antivitamines $\mathrm{K}$ en ville et en milieu hospitalier - (GEHT, HAS avril 2008).

*** Arrêt AVK : arrêter l'AVK à 4 à 5 jours avant l'intervention, reprise de l'AVK le soir ou le lendemain de l'intervention, pratiquer un INR à 48h (HAS 2008)

**** Arrêt AVK et relais par héparine : à J-5 arrêt de l'AVK, à J-3 relais par HBPM (ou HNF) à dose curative, à J-1 dernière injection HPBM le matin, HNF le soir, à JO intervention, à J+1 reprise AVK et héparine (à moduler en fonction du risque hémorragique), arrêt de l'héparine dès que l'INR cible est atteint 
Annexe IV. Algorithme systématique de prise en charge des patients sous anticoagulants oraux directs (AOD) en chirugie orale.

\section{Patients sous anticoagulants oraux directs}

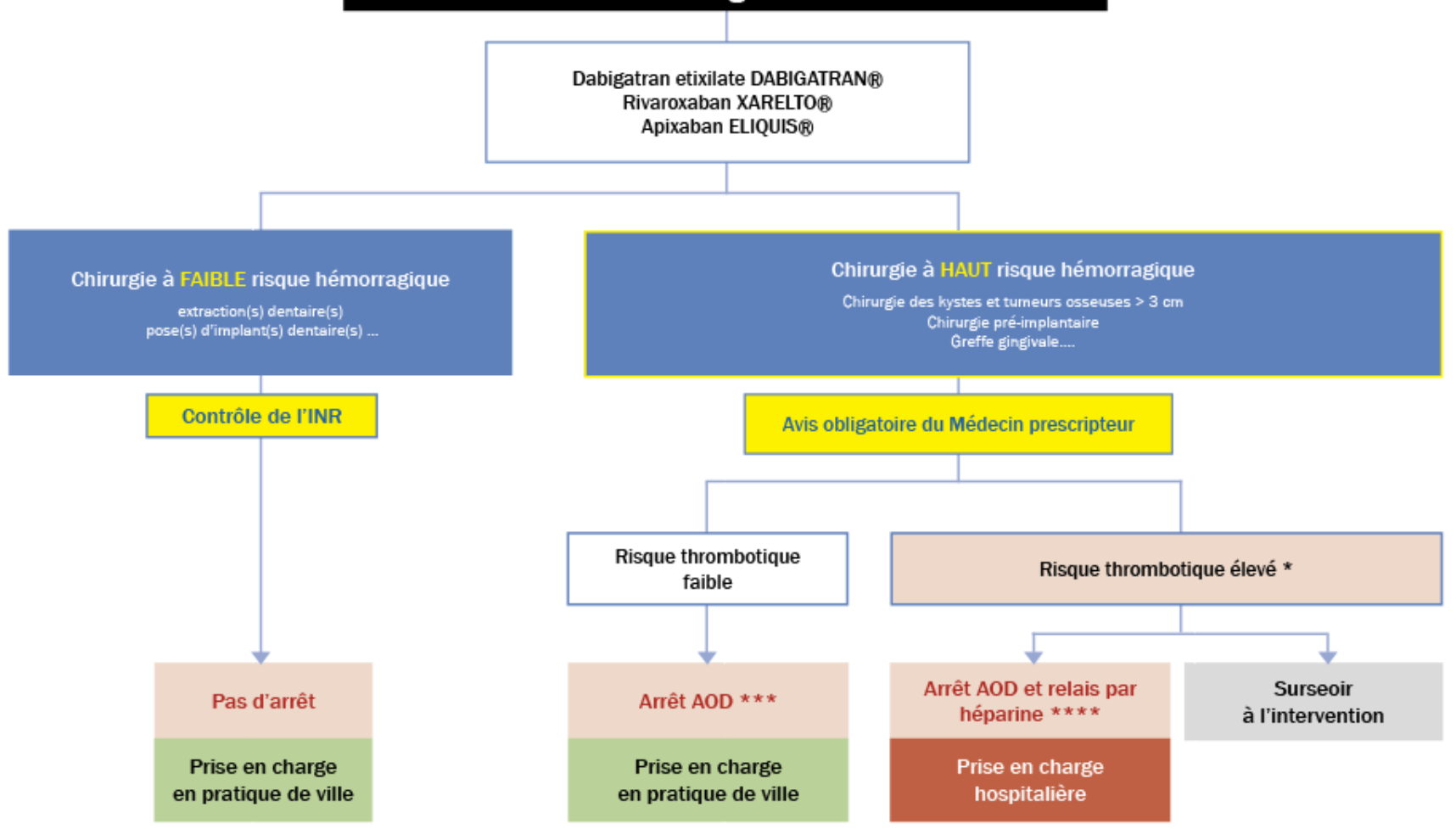

* Pas d'arrêt : il importe de préciser l'âge, l'indication (schéma curatif ou prévention en chirurgie orthopédique), la dose, le nombre de prises par jour, l'heure de la dernière de prise, geste à réaliser dans la demi-journée qui précède la prochaine prise

** Arrêt AOD : arrêter l'AOD la veille et le jour de l'intervention (fenêtre thérapeutique de 48h).

*** Arrêt AOD et relais par l'héparine : arrêter l'AOD 5 jours avant l'intervention chirurgicale. 
Annexe V. Algorithme systématique de prise en charge des patients sous héparines en chirugie orale.

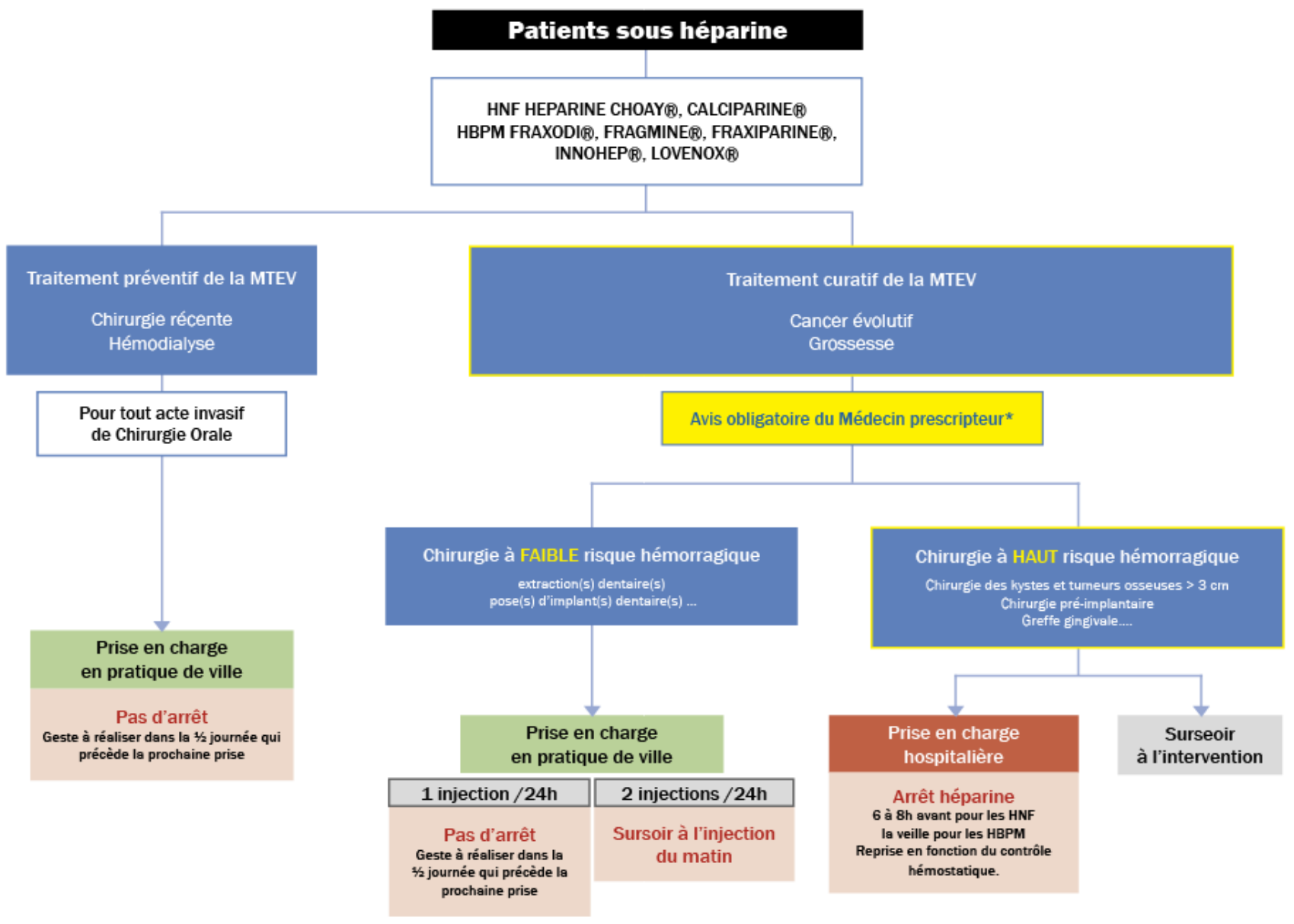

* vérifier si pas d'autres risques et précaution. 